\title{
Long-term effects of intravitreal bevacizumab and aflibercept on intraocular pressure in wet age-related macular degeneration
}

\author{
Mikael Kähkönen ${ }^{1,2}$, Raimo Tuuminen ${ }^{3,4}$ and Vesa Aaltonen ${ }^{1,2^{*}}$
}

\begin{abstract}
Background: To evaluate the incidence of sustained elevation of intraocular pressure (SE-IOP) associated with intravitreal injections of anti-vascular endothelial growth factors (anti-VEGF) bevacizumab and aflibercept in patients with wet age-related macular degeneration (WAMD).

Methods: A retrospective cohort study consisting of 120 eyes from 120 patients with anti-VEGF treatment for WAMD. Three different anti-VEGF groups were considered: i) 71 cases receiving bevacizumab only, ii) 49 cases receiving bevacizumab before switch to aflibercept, iii) 49 cases after switch to aflibercept. 120 uninjected fellow eyes served as controls. SE-IOP was defined as an increase from baseline $\geq 5 \mathrm{mmHg}$ on 2 consecutive follow-up visits. The incidence of SE-IOP was analysed using exact Poisson tests and survival analysis. The time course of IOP was evaluated with linear mixed effect modelling.

Results: In total, 6 treated eyes (2.38\% incidence per eye-year) and 9 fellow eyes (3.58\% incidence per eye-year) developed SE-IOP, and survival analysis showed no statistically significant difference $(p=0.43)$. Furthermore, the incidence of SE-IOP did not differ between the three anti-VEGF groups. Comparing the injected eyes of patients under 70 years to those of patients over 70 years, there was a statistically significant difference in survival without SE-IOP (incidence of $16.7 \%$ vs $0.7 \%$, respectively, $p<0.0001$ ).
\end{abstract}

Conclusion: Intravitreal anti-VEGF injections were not associated with sustained elevation of IOP. These results do not support the claim that repeated anti-VEGF injections could elevate IOP.

Keywords: Wet age-related macular degeneration, Anti-vascular endothelial growth factor, Bevacizumab, Aflibercept, Intraocular pressure

\footnotetext{
* Correspondence: vesa.aaltonen@tyks.fi

'Department of Ophthalmology, Turku University Hospital, PO Box 52, 20521

Turku, Finland

${ }^{2}$ Department of Ophthalmology, University of Turku, Turku, Finland

Full list of author information is available at the end of the article
}

(c) The Author(s). 2021 Open Access This article is licensed under a Creative Commons Attribution 4.0 International License, which permits use, sharing, adaptation, distribution and reproduction in any medium or format, as long as you give appropriate credit to the original author(s) and the source, provide a link to the Creative Commons licence, and indicate if changes were made. The images or other third party material in this article are included in the article's Creative Commons licence, unless indicated otherwise in a credit line to the material. If material is not included in the article's Creative Commons licence and your intended use is not permitted by statutory regulation or exceeds the permitted use, you will need to obtain permission directly from the copyright holder. To view a copy of this licence, visit http://creativecommons.org/licenses/by/4.0/ The Creative Commons Public Domain Dedication waiver (http://creativecommons.org/publicdomain/zero/1.0/) applies to the data made available in this article, unless otherwise stated in a credit line to the data. 


\section{Background}

Age-related macular degeneration (AMD) remains the leading cause of irreversible blindness in people aged over 50 in western countries, the wet form (wAMD) being responsible for approximately 90\% of blindness attributable to AMD [1]. The incidence of blindness from AMD has fallen significantly after the introduction of intravitreal injections of anti-vascular endothelial growth factors (anti-VEGF) for the treatment of wAMD [2, 3].

With the increasing number of patients receiving antiVEGF therapy for extended periods [4], it is important to evaluate the long-term adverse effects of the available injections. Anti-VEGFs bevacizumab and aflibercept are both safe and effective in treating wAMD. Adverse ocular events are rare and include, for example, increased intraocular pressure (IOP), retinal detachment and endophthalmitis [5].

IOP spikes may occur after various intraocular procedures, and precautions are particularly important in patients with glaucoma [6-8]. The transient IOP elevation after anti-VEGF injections was first described by Hollands et al., and the phenomenon is well known today. In most people, IOP and mean ocular perfusion pressure recover in $30 \mathrm{~min}$ after the injection $[9,10]$.

Conversely, the long-term effects of anti-VEGF agents on IOP are debated. A report from the IRIS (Intelligent Research in Sight) Registry revealed a clinically and statistically significant sustained IOP rise overall and specifically in eyes receiving bevacizumab or ranibizumab, but not in eyes that received aflibercept [11]. The overall findings of the registry have been backed by multiple smaller studies [12-14].

In contrast, several reports have concluded that antiVEGF therapies do not have a significant long-term effect on IOP [15-18]. An analysis of the VIEW (VEGF Trap-Eye: Investigation of Efficacy and Safety in Wet AMD) 1 and 2 studies showed a slight decrease in mean IOP in eyes receiving intravitreal aflibercept for 96 weeks while there was no such decrease in the ranibizumab group. The study also demonstrated a significant decrease in the incidence of elevated IOP when comparing aflibercept to ranibizumab [19]. In a recent metaanalysis of 46 studies investigating intravitreal bevacizumab, ranibizumab and aflibercept, IOP normalized 1 week after the injection and no significant change in IOP was found for longer time-intervals [20]. It remains inconclusive whether intravitreal anti-VEGF therapy leads to an elevated risk of sustained IOP rise.

The purpose of this real-world study was to evaluate the incidence of sustained elevation of IOP (SE-IOP) and the IOP kinetics in WAMD patients that were treated either with bevacizumab only or switched to aflibercept.

\section{Methods}

\section{Study design and patients}

This retrospective cohort study included 120 eyes from 120 patients with intravitreal anti-VEGF treatment for wAMD and 120 fellow eyes which were not treated with any intravitreal anti-VEGF agent (controls). Of the treated eyes, 71 received bevacizumab only (nonswitched patients), while 49 received bevacizumab as first-line treatment and were later switched to aflibercept as per the treatment protocol (switched patients). Three study groups were formed: i) 71 patients receiving bevacizumab only, ii) 49 patients receiving bevacizumab before switch to aflibercept, iii) the same 49 patients receiving aflibercept after the switch.

The patients were treated at Turku University Hospital between June 2011 and July 2019, and they were identified through the corresponding diagnosis (International Classification of Diseases [ICD]: diagnosis H35.31 and procedure code CKD05). Patients were included in this study if they received bevacizumab only or bevacizumab and aflibercept consecutively in one eye for wAMD and they were anti-VEGF treatment-naive in both eyes. The study followed the guidelines of the Declaration of Helsinki.

Patient records and prescription databases were searched for possible confounding conditions (established glaucoma and ocular hypertension), medications (IOP-lowering drugs) and treatments (cataract surgery, glaucoma surgery, iridotomy and laser trabeculoplasty). In the study, the definition of glaucoma was based on ICD coding: eyes were treated as glaucomatous if they were associated with any sub-code under the main code $\mathrm{H} 40$, which includes established glaucoma as well as ocular hypertension.

\section{Exclusion criteria}

Exclusion criteria were ongoing anti-VEGF treatment in the fellow eye, less than two follow-up visits in any study group, lack of meaningful IOP measurements in the fellow eye (e.g. ocular prosthesis, phthisis bulbi) and lack of a baseline IOP measurement in either eye. During the follow-up, if intravitreal anti-VEGF treatment was initiated in the fellow eye, or the patient dropped out of either the bevacizumab or aflibercept treatment protocol, data was included up to this event.

\section{Clinical evaluation}

The patients were diagnosed with wAMD by typical findings in dilated fundoscopy and optical coherence tomography (OCT) (Heidelberg Spectralis, Heidelberg Engineering $\mathrm{GmbH}$, Heidelberg, Germany): cystic macular edema (CME) and/or neuroepithelial detachment (NED) and/or pigment epithelial detachment (PED) and/ or retinal hemorrhage. Fluorescein angiography was performed in cases when needed in differential diagnosis. 
Each visit included visual acuity testing and tonometry. Rebound tonometry (iCare ${ }^{\circ}$ tonometer, Revenio Group, Vantaa, Finland) was the standard practice. Single measurements from alternate methods were excluded for the consistency of IOP data.

\section{Bevacizumab treatment protocol}

Treatment of all patients started with three monthly injections of bevacizumab (induction phase), followed by a control visit 1 month after the last injection. If the patient had full response to bevacizumab (no CME, no NED, no new hemorrhage, no PED increase), the treatment was continued with three subsequent injections of bevacizumab with 2 -month (8 weeks) intervals. The patients were controlled after the first 2-month interval with OCT, and later, 2 months after each series of three bevacizumab injections (visual acuity, tonometry, dilated fundoscopy and OCT). Any CME, NED, new hemorrhage and PED increase was considered activity. If activity was observed in any control, the injection interval was tapered between 4 and 8 weeks, depending on the observed activity and response. The treatment always continued for a minimum of 15 months, followed by a 12-month observation period.

\section{Aflibercept treatment protocol}

If a sufficient response was not achieved with six monthly bevacizumab injections, the treatment was switched to aflibercept modified treat-and-extend regimen (TER) protocol. In the aflibercept modified TER protocol, the first injection interval was 4 weeks, and subsequent injection intervals were increased by 2 weeks when no activity was observed (no CME, no NED, no new hemorrhage, no PED increase). If any activity was observed, the injection interval was decreased by 1 week. If activity was observed repeatedly ( 2 times) with the same injection interval, the longest interval with which there was no activity, was kept for a period of two consecutive injection series and then extended again.

Next injection interval was decided based on the OCT findings during each injection visit. Follow-up visits were planned as follows: after three injections with 4-8-week intervals; after two injections with 9-12-week intervals; after one injection with 13-16-week interval.

\section{Definition of SE-IOP}

SE-IOP was defined as an increase from baseline $\geq 5$ $\mathrm{mmHg}$ on at least 2 consecutive follow-up visits. The criterion was chosen to take into account variation in baseline IOP and to exclude non-sustained elevation of IOP.

\section{Statistical analysis}

Descriptive statistics for continuous variables are presented using mean and standard deviation (SD) when variable is normally distributed, and median with first and third quartiles (Q1, Q3) otherwise. For categorical variables, frequencies and percentages are used. Normality of variables was assessed with Shapiro-Wilk test. Baseline characteristics between the three study groups were tested for statistically significant differences using analysis of variance (ANOVA) for normally distributed and Kruskal-Wallis test for non-normally distributed continuous variables. Chi-square test was used for categorical variables. For some of the following analyses, the groups were combined to increase the statistical power.

To investigate the rate of SE-IOP, eye-year incidence rates with confidence intervals were calculated with exact Poisson tests. Only the time to first event was considered. Corresponding exact mid-p adjusted $p$-values are also presented. A generalised linear mixed effects model was created to assess the clinical predictors for SE-IOP. The interaction between each potential predictor and the treatment status of the eye (whether it was a treated or a fellow eye) was analysed first separately and then all statistically significant interactions were combined in a multivariate model. Additionally, the Kaplan-Meier method was used to estimate survival curves, and comparisons were made between the treated and fellow eyes, and between the treated eyes of two age groups ( $<70$ and $\geq 70$ years) using log-rank test.

In addition to the incidence of SE-IOP, the time course of IOP was analysed. Time from the beginning of treatment was converted to a categorical variable. To compare the time course of IOP between the treated and fellow eyes and between the three study groups, linear mixed effect models were created. Least squares means were obtained from the models and plotted, and the interactions between time from baseline and predictor variables (treated versus fellow eyes, study groups versus each other) were analysed using ANOVA.

Two-sided tests were used in all statistical analyses and $p$-values $<0.05$ were considered statistically significant. Statistical analysis was performed using R version 4.0.1 ( $\mathrm{R}$ Foundation for Statistical Computing, Vienna, Austria).

\section{Results}

\section{Patient characteristics}

Patient characteristics are summarized in Table 1. Between the groups, switched patients on aflibercept had a significantly longer follow-up and a higher cumulative number of injections compared to the other two groups. Non-switched patients on bevacizumab had a longer treatment interval compared to the other two groups. There were no 
Table 1 Patient characteristics

\begin{tabular}{|c|c|c|c|c|}
\hline & Non-switched patients, $n=71$ & Switched patier & & \\
\hline & Bevacizumab & Bevacizumab $^{a}$ & Aflibercept $^{a}$ & $p$-value \\
\hline Sex (males), n (\%) & $29(41)$ & $15(31)$ & $15(31)$ & 0.39 \\
\hline Age at first injection (years), mean \pm SD & $78.3 \pm 7.6$ & $76.7 \pm 8.0$ & $78.0 \pm 7.9$ & 0.51 \\
\hline Glaucoma, n (\%) & & & & \\
\hline Treated eye & $3(4)$ & $5(10)$ & $5(10)$ & 0.36 \\
\hline Fellow eye & $4(6)$ & $5(10)$ & $5(10)$ & 0.57 \\
\hline Pseudophakia, n (\%) & & & & \\
\hline Treated eye & $33(46)$ & $15(31)$ & $23(47)$ & 0.16 \\
\hline Fellow eye & $31(44)$ & $17(35)$ & $21(43)$ & 0.58 \\
\hline Baseline IOP (mmHg), median (Q1; Q3) & & & & \\
\hline Treated eye & $13(12 ; 16)$ & $13(10 ; 15)$ & $13(11 ; 15)$ & 0.85 \\
\hline Fellow eye & $14(11 ; 16)$ & $12(11 ; 16)$ & $14(10 ; 17)$ & 0.82 \\
\hline Last IOP (mmHg), median (Q1; Q3) & & & & \\
\hline Treated eye & $13(11 ; 15)$ & $13(11 ; 15)$ & $12(10 ; 14)$ & 0.069 \\
\hline Fellow eye & $12(10 ; 15)$ & $14(10 ; 17)$ & $13(10 ; 15)$ & 0.26 \\
\hline Follow-up (days), mean \pm SD & $495.8 \pm 245.2$ & $494.5 \pm 351.4$ & $700.2 \pm 331.0$ & $<0.001$ \\
\hline No. of injections, median (Q1; Q3) & $10(9 ; 14.5)$ & $9(6 ; 15)$ & $15(12 ; 21)$ & $<0.001$ \\
\hline Injection interval (days), median (Q1; Q3) & $41.7(34.2 ; 49.0)$ & $36.5(34.0 ; 40.3)$ & $35.1(30.4 ; 55.5)$ & 0.008 \\
\hline
\end{tabular}

${ }^{\mathrm{a}}$ Same individuals

statistically significant differences in baseline or last IOP between treated and fellow eyes (Table 1).

During the follow-up, glaucoma treatment was enhanced for one patient with bilateral selective laser trabeculoplasty and an additional IOP-lowering drug for the treated eye (Table 1, switched patients, bevacizumab). Another patient was treated for bilateral narrow-angle glaucoma with an IOP-lowering drug bilaterally, followed by a bilateral laser iridotomy (Table 1, switched patients, bevacizumab). This was the only patient diagnosed with glaucoma newly during the follow-up. None of the patients were subjected to glaucoma surgery. Patients without glaucoma did not use any IOPlowering drugs or receive any other IOP-lowering treatments during the follow-up.

Incidence of sustained elevation of intraocular pressure Table 2 represents the eye-year incidence in each group and the difference between treated and fellow eyes. The

Table 2 Incidence of SE-IOP

\begin{tabular}{|c|c|c|c|c|}
\hline & Number of eyes & Eyes with SE-IOP & Eye-year incidence, \% $(95 \% \mathrm{Cl})$ & $p$-value \\
\hline \multicolumn{5}{|c|}{ Non-switched patients } \\
\hline \multicolumn{5}{|l|}{ Bevacizumab } \\
\hline Treated eye & 71 & 3 & $3.17(0.65 ; 9.26)$ & 0.69 \\
\hline Fellow eye & 71 & 2 & $2.11(0.26 ; 7.63)$ & ref \\
\hline \multicolumn{5}{|l|}{ Switched patients } \\
\hline \multicolumn{5}{|l|}{ Bevacizumab } \\
\hline Treated eye & 49 & 2 & $3.09(0.37 ; 11.18)$ & 0.46 \\
\hline Fellow eye & 49 & 4 & $6.17(1.68 ; 15.79)$ & ref \\
\hline \multicolumn{5}{|l|}{ Aflibercept } \\
\hline Treated eye & 49 & 1 & $1.07(0.03 ; 5.98)$ & 0.37 \\
\hline Fellow eye & 49 & 3 & $3.28(0.68 ; 9.57)$ & ref \\
\hline \multicolumn{5}{|l|}{ All study groups } \\
\hline Treated eye & 169 & 6 & $2.38(0.87 ; 5.17)$ & 0.45 \\
\hline Fellow eye & 169 & 9 & $3.58(1.64 ; 6.8)$ & ref \\
\hline
\end{tabular}


incidence of SE-IOP was comparable between treated and fellow eyes among patients on bevacizumab only, and switched patients both during bevacizumab and during aflibercept administration (Table 2). Furthermore, using the treated eye of non-switched patients on bevacizumab as a reference, the treated eye of neither switched patients on bevacizumab ( $p=1.00$, Table 2$)$ nor switched patients on aflibercept ( $p=0.38$, Table 2$)$ showed a statistically significant difference in the eye-year incidence of SE-IOP.

In total, 6 treated eyes (2.38\% incidence per eye-year) and 9 fellow eyes (3.58\%) developed SE-IOP, the difference being statistically non-significant $(p=0.45$, Table 2$)$. To illustrate the incidence of SE-IOP in treated and fellow eyes, Kaplan-Meier curves were drawn, showing no association between the development of SE-IOP and the treatment status of the eye $(p=0.43$, Fig. $1 \mathrm{~A})$. Additionally, a generalized linear mixed effects model was created to assess the clinical predictors for SE-IOP during anti-VEGF therapy. All anti-VEGF groups were pooled together. The model revealed an inverse association between age and incidence of SE-IOP, younger age being a statistically significant risk factor $(p=0.018)$. In the univariate models, longer anti-VEGF treatment duration also appeared to be a risk factor $(p=0.036)$, but it was no longer statistically significant in the multivariate model $(p=0.140)$, whereas younger age remained a statistically significant risk factor $(p=0.016)$. A Kaplan-Meier curve is provided, showing this association $(p<0.0001$, Fig. 1B). Sex, glaucoma, phakic status, baseline IOP, total number of injections and injection interval were not associated with SE-IOP during anti-VEGF therapy (data not shown).

\section{Time course of intraocular pressure}

Figure 2 demonstrates changes in IOP during follow-up. There were no differences in the time course of IOP between treated and fellow eyes in any group (Fig. 2A-C).
Additionally, to illustrate the differences between the three groups, a line chart including only the anti-VEGF treated eyes from each group is provided. When comparing these treated eyes between the groups, a statistically significant difference was observed in the time course of IOP $(p=$ 0.033 , Fig. 2D). While IOP remained relatively constant in non-switched patients on bevacizumab, there was a slight increase in IOP in switched patients receiving bevacizumab and a slight decrease in switched patients on aflibercept. A comparison between switched patients on bevacizumab and switched patients on aflibercept showed statistical significance ( $p=0.015$, Fig. 2D).

\section{Discussion}

The purpose of this study was to evaluate the incidence of SE-IOP and the time course of IOP in eyes receiving bevacizumab only or aflibercept after insufficient bevacizumab treatment response, and in their fellow eyes, in a real-life setting. A strict treatment protocol was followed, and only patients with unilateral wAMD were included. Pooling all study groups together, our results show a low eye-year incidence for SE-IOP, at $2.38 \%$ in treated eyes and $3.58 \%$ in fellow eyes. This incidence is in line with previous studies using similar criteria for SE-IOP [21].

Previous studies have had conflicting results on the long-term effects of anti-VEGFs on IOP. Reis et al. identified a statistically significant rise in the incidence of SE-IOP when comparing injected eyes to control eyes (7.47\% versus $0.93 \%$ ) [13]. Another controlled study by Wehrli et al. reported a $0.51 \%$ eye-year incidence in the treated eye and $1.00 \%$ in the fellow eye, a statistically non-significant difference [15]. Our results are in accordance with their conclusions that the difference in the incidence of SE-IOP between treated and fellow eyes is non-significant.
(A)

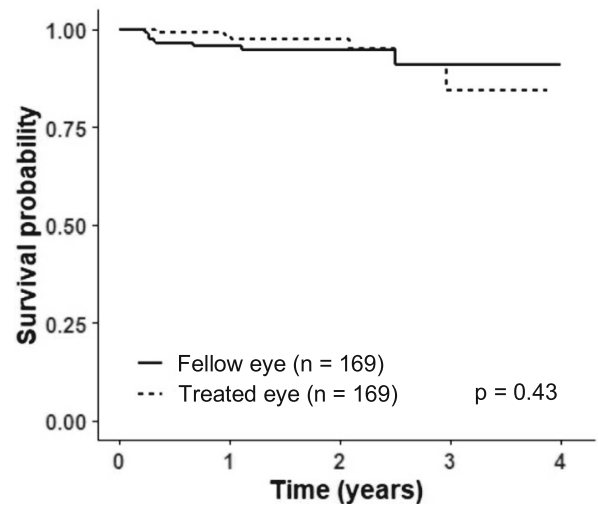

(B)

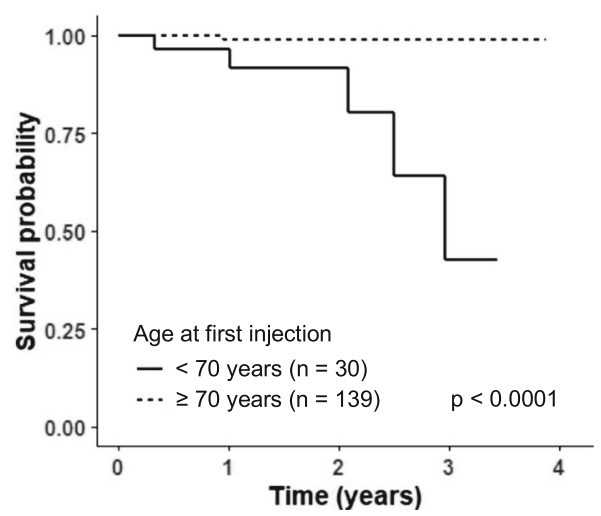

Fig. 1 Kaplan-Meier survival curves for (A) SE-IOP in treated eyes versus fellow eyes and (B) in treated eyes of patients under 70 years versus those over 70 years. All study groups are pooled together. P-values were calculated with log-rank test 
(A)

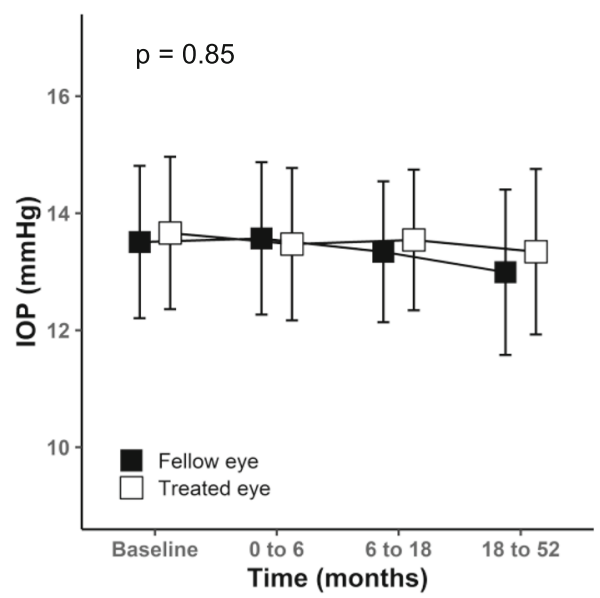

(C)

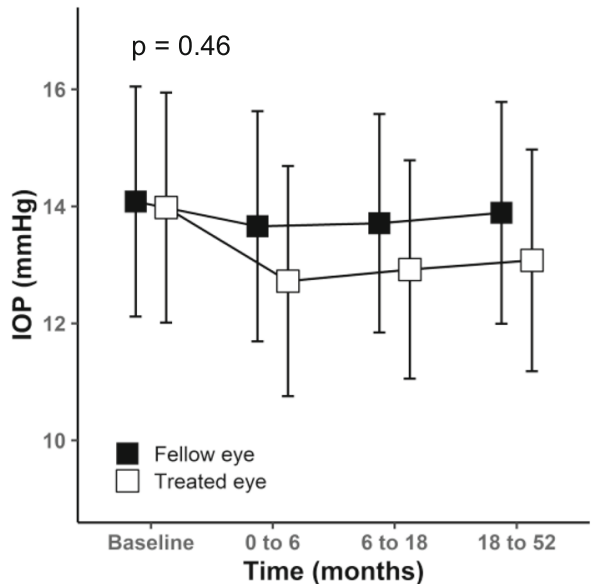

(B)

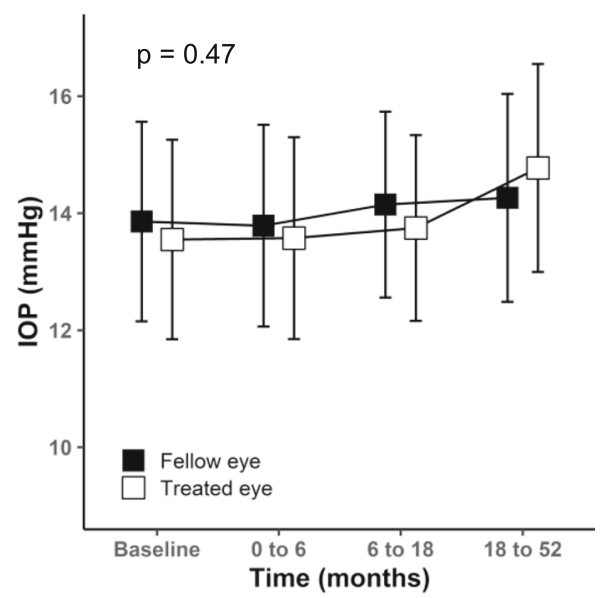

(D)

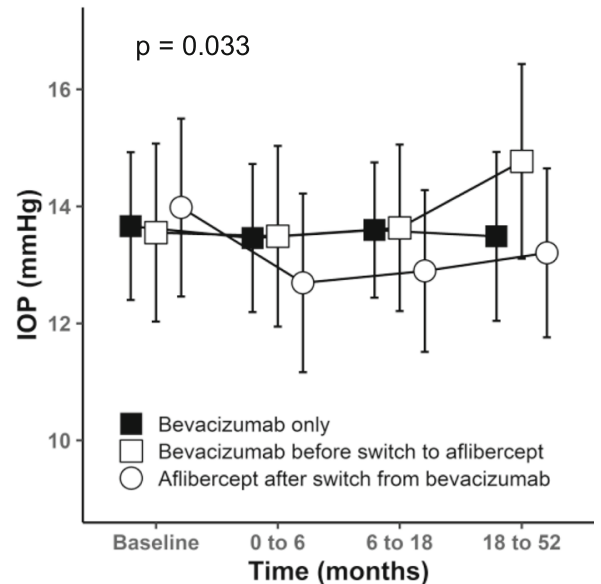

Fig. 2 The time course of IOP $(\mathbf{A})$ in treated versus fellow eyes during bevacizumab in non-switched patients, (B) bevacizumab in switched patients, $(\mathbf{C})$ aflibercept in switched patients, and (D) in treated eyes only, comparing the three study groups. Plotted IOP data represent least squares means with $95 \%$ confidence intervals. Reported p-values describe the statistical significance of the interaction between time from baseline and treatment status $(\mathbf{A}-\mathbf{C})$, and time from baseline and study group (D)

Reis et al. and Bilgic et al. showed that the incidence of SE-IOP is associated with older age and pre-existing glaucoma among other clinical predictors [12, 13]. Various potential predictors were analysed in our study, including glaucoma status, baseline IOP, sex and injection interval, but only younger age was determined to be a risk factor for SE-IOP associated with anti-VEGF therapy. This might partly be explained by a slight positive correlation between age and injection interval in our sample, younger people receiving more intensive therapy. However, injection interval itself was determined to not be associated with the incidence of SE-IOP. Younger patients also had a slightly higher baseline IOP, which itself showed a weak association with higher incidence of
SE-IOP in the univariate model, while not quite being statistically significant. It should be noted that this effect might have been suppressed by some of the eyes with higher baseline IOP regressing towards the mean as the baseline only consisted of one measurement per patient.

Glaucoma treatment was changed bilaterally for two patients during the follow-up. Of these treatment modifications, one was symmetrical and one asymmetrical between the treated and fellow eyes. Therefore, the glaucoma treatment modifications are not likely to have any significant confounding effect. Furthermore, as the treatment for other glaucoma patients remained unchanged during the follow-up, and only patients with glaucoma or ocular hypertension received any IOP- 
lowering treatments, we conclude that IOP-lowering treatments are not significant confounders.

Our results are in line with previous reports in that bevacizumab seems to cause slightly higher IOP values than aflibercept $[11,18,22]$. The findings of Freund et al. demonstrating a slight decrease in IOP in eyes receiving aflibercept [19] are also supported by the present study. The reason for this favourable effect of aflibercept remains unknown. Unlike bevacizumab that only binds VEGF-A, aflibercept traps other VEGF family members too, including VEGF-B and placental growth factor (PlGF). Moreover, it has a markedly higher affinity for VEGF-A [23]. VEGF receptors expressed in the human trabecular meshwork tissue [24] could mediate unknown effects of VEGF-B and PIGF. It has also been hypothesized that silicone oil and protein particles found in antiVEGF drugs might obstruct the outflow pathway [25], and this effect could in theory differ between the drugs. The researchers concluded that while bevacizumab and aflibercept are similar in average product quality, repackaged bevacizumab does show varying levels of contamination. Thirdly, repeated transient IOP spikes after injections might predispose the eye to SE-IOP via damaging the trabecular meshwork [26], although this cannot yet explain why aflibercept seems less harmful as no studies have demonstrated a statistically significant difference in the short-term course of IOP after an injection with bevacizumab versus aflibercept. More research comparing the pharmacodynamics of these two drugs are needed.

A notable limitation of this study was that the patients received aflibercept only after attempted therapy with bevacizumab. Because of this, it is possible that some of the effects seemingly caused by aflibercept resulted instead from the discontinuing of bevacizumab. However, the difference between the drugs remains. Additionally, the relatively small sample size may have decreased the ability to detect statistically significant effects some other researchers have demonstrated. The known systemic effects of intravitreal anti-VEGF injections [27] could also explain why no differences between the treated and fellow eyes were observed as the fellow eye might not be a true control.

\section{Conclusion}

To summarize, our results show a low incidence of SEIOP during anti-VEGF treatment, and no IOP-related effects attributable to the injections were seen. Younger patients might benefit from a closer follow-up of IOP. Some of these results conflict with past literature, and further controlled studies with larger sample sizes are still needed to investigate the possible long-term effects intravitreal anti-VEGF injections have on IOP.

\begin{abstract}
Abbreviations
AMD: Age-related macular degeneration; Anti-VEGF: Anti-vascular endothelial growth factor; CME: Cystic macular edema; ICD: International Classification of Diseases; IOP: Intraocular pressure; NED: Neuroepithelial detachment; OCT: Optical coherence tomography; PIGF: Placental growth factor; PED: Pigment epithelial detachment; SE-IOP: Sustained elevation of intraocular pressure; TER: Treat-and-extend regimen; WAMD: Wet age-related macular degeneration
\end{abstract}

\section{Acknowledgements \\ We would like to thank biostatistician ms. Helena Ollila from the University of Turku for her great help in statistical analysis.}

\section{Authors' contributions}

MK: Conception of the study, data acquisition and analysis, interpretation of the data and writing the manuscript. RT: Designing the work, interpretation of the data and substantive revision of the manuscript. VA: Conception of the study, data acquisition, interpretation of the data and writing the manuscript. All authors have read and approved the final manuscript.

\section{Funding}

The study was supported by grants from the University of Turku. The funding body had no role in the design or conduct of this research.

\section{Availability of data and materials}

The datasets analysed during the current study are not publicly available in order to protect patient identity and confidentiality, but they are available from the corresponding author on reasonable request.

\section{Declarations}

Ethics approval and consent to participate

Ethics committee of Turku University Hospital waived the need for formal approval in this case since no ethical assessment or approval by an independent review board or obtaining consent of the patients is mandatory for a registry study in Finland (Medical Research Act 1999/488, revised by 2004/295 and 2010/794).

\section{Consent for publication}

Not applicable.

\section{Competing interests}

VA has received fees for consultation from Roche and for consultation and lectures from Bayer and Novartis outside of this work. RT has received fees for consultation and lectures from Alcon, Bayer and Novartis outside of this work.

\section{Author details}

${ }^{1}$ Department of Ophthalmology, Turku University Hospital, PO Box 52, 20521 Turku, Finland. 'Department of Ophthalmology, University of Turku, Turku, Finland. ${ }^{3}$ Helsinki Retina Research Group, Faculty of Medicine, University of Helsinki, Helsinki, Finland. ${ }^{4}$ Department of Ophthalmology, Helsinki University Hospital, Helsinki, Finland.

Received: 7 January 2021 Accepted: 14 August 2021

Published online: 28 August 2021

\section{References}

1. Chappelow AV, Schachat AP. Neovascular age-related macular degeneration. In: Nguyen Q, Rodrigues E, Farah M, Mieler WF, editors. Retinal pharmacotherapy. Philadelphia: Saunders; 2010. p. 128-32. https:// doi.org/10.1016/B978-1-4377-0603-1.00023-5.

2. Bloch SB, Larsen M, Munch IC. Incidence of Legal Blindness From AgeRelated Macular Degeneration in Denmark: Year 2000 to 2010. Am J Ophthalmol. 2012;153:209-213.e2.

3. Jonas JB, Cheung CMG, Panda-Jonas S. Updates on the Epidemiology of Age-Related Macular Degeneration. Asia Pac J Ophthalmol (Phila). 2017;6: 493-7.

4. Baek SK, Kim JH, Kim JW, Kim CG. Increase in the population of patients with Neovascular age-related macular degeneration who underwent longterm active treatment. Sci Rep. 2019;9:1-10. 
5. Bakri SJ, Thorne JE, Ho AC, Ehlers JP, Schoenberger SD, Yeh S, et al. Safety and efficacy of anti-vascular endothelial growth factor therapies for Neovascular age-related macular degeneration: a report by the American Academy of ophthalmology. Ophthalmology. 2019;126(1):55-63. https://doi. org/10.1016/j.ophtha.2018.07.028.

6. Grzybowski A, Kanclerz P. Early postoperative intraocular pressure elevation following cataract surgery. Curr Opin Ophthalmol. 2019;30(1):56-62. https:// doi.org/10.1097/ICU.0000000000000545.

7. Abedi G, Adelman RA, Salim S. Incidence and management of elevated intraocular pressure with antivascular endothelial growth factor agents. Semin Ophthalmol. 2013;28(3):126-30. https://doi.org/10.3109/08820538.2 013.771195.

8. Achiron A. Intraocular pressure spikes following neodymium-doped yttrium aluminum garnet laser capsulotomy: current prevalence and Management in Israel. J Curr Glaucoma Pract. 2017;11(2):63-6. https://doi.org/10.5005/jpjournals-10028-1225.

9. Hollands H, Wong J, Bruen R, Campbell RJ, Sharma S, Gale J. Short-term intraocular pressure changes after intravitreal injection of bevacizumab. Can J Ophthalmol. 2007;42(6):807-11. https://doi.org/10.3129/i07-172.

10. Lee JW, Park H, Choi JH, Lee HJ, Moon SW, Kang JH, et al. Short-term changes of intraocular pressure and ocular perfusion pressure after intravitreal injection of bevacizumab or ranibizumab. BMC Ophthalmol. 2016;16(1):69. https://doi.org/10.1186/s12886-016-0255-8.

11. Atchison EA, Wood KM, Mattox CG, Barry CN, Lum F, MacCumber MW. The real-world effect of Intravitreous anti-vascular endothelial growth factor drugs on intraocular pressure: an analysis using the IRIS registry. Ophthalmology. 2018;125(5):676-82. https://doi.org/10.1016/j.ophtha.201 7.11.027.

12. Bilgic A, Kodjikian L, Chhablani J, Sudhalkar A, Trivedi M, Vasavada V, et al. Sustained intraocular pressure rise after the treat and extend regimen at 3 years: Aflibercept versus Ranibizumab. J Ophthalmol. 2020;2020:1-8.

13. Reis GM, Grigg J, Chua B, Lee A, Lim R, Higgins R, et al. Incidence of intraocular pressure elevation following intravitreal Ranibizumab (Lucentis) for age-related macular degeneration. J Curr Glaucoma Pract. 2017;11(1):3-7. https://doi.org/10.5005/jp-journals-10008-1213.

14. Beato J, Pedrosa AC, Pinheiro-Costa J, Freitas-da-Costa P, Falcão MS, Melo A, et al. Long-term effect of anti-VEGF agents on intraocular pressure in agerelated macular degeneration. Ophthalmic Res. 2016;56(1):30-4. https://doi. org/10.1159/000444395.

15. Wehrli SJ, Tawse K, Levin MH, Zaidi A, Pistilli M, Brucker AJ. A lack of delayed intraocular pressure elevation in patients treated with intravitreal injection of bevacizumab and ranibizumab. Retina. 2012;32(7):1295-301. https://doi. org/10.1097/IAE.0b013e31823f0c95.

16. Kim D, Nam W, Kim H, Yi K. Does intravitreal injections of bevacizumab for age-related macular degeneration affect long-term intraocular pressure? J Glaucoma. 2014;23(7):446-8. https://doi.org/10.1097/JJG.0b013e3182946505.

17. Nariani A, Williams B, Hariprasad S. Long-term effect of anti-vascular endothelial growth factor injections on intraocular pressure. Indian 1 Ophthalmol. 2016;64(9):643-7. https://doi.org/10.4103/0301-4738.194329.

18. Moraru A, Pînzaru G, Moțoc A, Costin D, Brănişteanu D. Incidence of ocular hypertension after intravitreal injection of anti-VEGF agents in the treatment of neovascular AMD. Rom J Ophthalmol. 2017;61(3):207-11. https://doi.org/1 0.22336/rjo.2017.38.

19. Freund KB, Hoang QV, Saroj N, Thompson D. Intraocular pressure in patients with Neovascular age-related macular degeneration receiving intravitreal Aflibercept or Ranibizumab. Ophthalmology. 2015;122(9):1802-10. https:// doi.org/10.1016/j.ophtha.2015.04.018.

20. de Vries VA, Bassil FL, Ramdas W. The effects of intravitreal injections on intraocular pressure and retinal nerve fiber layer: a systematic review and meta-analysis. Sci Rep. 2020;10(1):13248. https://doi.org/10.1038/s41598-02070269-7.

21. Zhou Y, Zhou M, Xia S, Jing Q, Gao L. Sustained elevation of intraocular pressure associated with intravitreal Administration of Anti-vascular Endothelial Growth Factor: a systematic review and Meta-analysis. Sci Rep. 2016;6:1-9.

22. Yannuzzi NA, Patel SN, Bhavsar KV, Sugiguchi F, Freund KB. Predictors of Sustained Intraocular Pressure Elevation in Eyes Receiving Intravitreal AntiVascular Endothelial Growth Factor Therapy. Am J Ophthalmol. 2014:158: 319-327.e2.

23. Papadopoulos N, Martin J, Ruan Q, Rafique A, Rosconi MP, Shi E, et al. Binding and neutralization of vascular endothelial growth factor (VEGF) and related ligands by VEGF trap, ranibizumab and bevacizumab. Angiogenesis. 2012;15(2):171-85. https://doi.org/10.1007/s10456-011-9249-6.

24. Fujimoto T, Inoue T, Maki K, Inoue-Mochita M, Tanihara H. Vascular endothelial growth factor-a increases the aqueous humor outflow facility. PLoS One. 2016;11(9):e0161332. https://doi.org/10.1371/journal.pone.01 61332.

25. Schargus M, Werner BP, Geerling G, Winter G. Contamination of anti-VEGF drugs for intravitreal injection: how do repackaging and newly developed syringes affect the amount of silicone oil droplets and protein aggregates? Retina. 2018;38(10):2088-95. https://doi.org/10.1097/IAE.0000000000001809.

26. Battista SA, Lu Z, Hofmann S, Freddo T, Overby DR, Gong H. Reduction of the available area for aqueous humor outflow and increase in meshwork Herniations into collector channels following acute IOP elevation in bovine eyes. Invest Ophthalmol Vis Sci. 2008:49(12):5346-52. https://doi.org/10.11 67/iovs.08-1707.

27. Carneiro ÂM, Costa R, Falcão MS, Barthelmes D, Mendonça LS, Fonseca SL, et al. Vascular endothelial growth factor plasma levels before and after treatment of neovascular age-related macular degeneration with bevacizumab or ranibizumab. Acta Ophthalmol. 2012;90(1):e25-30. https:// doi.org/10.1111/j.1755-3768.2011.02240.x.

\section{Publisher's Note}

Springer Nature remains neutral with regard to jurisdictional claims in published maps and institutional affiliations.

\section{Ready to submit your research? Choose BMC and benefit from:}

- fast, convenient online submission

- thorough peer review by experienced researchers in your field

- rapid publication on acceptance

- support for research data, including large and complex data types

- gold Open Access which fosters wider collaboration and increased citations

- maximum visibility for your research: over $100 \mathrm{M}$ website views per year

At BMC, research is always in progress.

Learn more biomedcentral.com/submissions 